\title{
Atopic Dermatitis (AD) Disease History With AD Treatment History in a Cohort of AD Patients Treated With Dupilumab From a Real-World Registry (PROSE)
}

Tien Q. Nguyen ${ }^{1}$, Hermenio Lima ${ }^{2}$, Lindsey Finklea ${ }^{3}$, Haixin Zhang ${ }^{4}$, Daniel Richman ${ }^{5}$, Andrew Korotzer ${ }^{4}$, Shikha Bansal ${ }^{4}$ 'First OC Dermatology, Fountain Valley, CA, USA; ${ }^{2}$ McMaster University, Hamilton, ON, Canada; ${ }^{3}$ RFSA Dermatology, San Antonio, TX, USA; ${ }^{4}$ Regeneron Pharmaceuticals, Inc., Tarrytown, NY, USA; ${ }^{5}$ Sanofi Genzyme, Cambridge, MA, USA

\section{BACKGROUND}

- Dupilumab is a fully human monoclonal antibody that blocks the shared receptor component for safety profile and sustained efficacy in adult and pediatric patients (aged $>6$ years) with moderate-to-severe atopic dermatitis (AD) $)^{3-6}$

There is currently a paucity of information on patients who receive dupilumab in a real-world setting

We present baseline data from a real-world registry of adult AD palients, intlating commercial dupilumab

\section{OBJECTIVE}

- To report patient and family history of $A D$, and AD treatments taken before treatment initiation with dupilumab in patients from the PROSE registry

\section{METHODS}

Study design

- PROSE (NCT03428646) is an ongoing (initiated: April 2018), multicenter, longitudinal, prospective, up-to-5characterizing dupilumab-treated $A D$ patients in a real-

Baseline data were recorded at the time of entry into the registry

Analysis

Data presented here are from the first interim analysis set of adult patients receiving dupilumab (data cutoff: July 2019)

All analyses are descriptive without imputation for missing values

\section{RESULTS}

Patients and demographics

315 patients were enrolled (Table 1) Approximately half of the patients were female, and $60 \%$ White Mean age of patients was 42.5 years and the mean duration of $A D$ was 19.7 years

Patient AD history

- $35 \%$ of patients had a family history of AD (Table 2) $54.0 \%$ of patients reported one or more type 2 inflammatory comorbidities in the 12 months prior to dupilumab initiation, including allergic rhinitis which was reported in $32.7 \%$ of patients (Table 3 )

Table 1. Baseline demographics and disease characteristics.

\begin{tabular}{|c|c|}
\hline & $\mathrm{N}=315$ \\
\hline Age, mean (SD), years & $42.5(16.99)$ \\
\hline Female sex, $n(\%)$ & $174(55.2)$ \\
\hline \multicolumn{2}{|l|}{ Race, $n(\%)$} \\
\hline White & $187(59.4)$ \\
\hline Black or African American & $56(17.8)$ \\
\hline Asian & $51(16.2)$ \\
\hline Other ${ }^{a}$ & $7(2.2)$ \\
\hline Not reported & $14(4.4)$ \\
\hline Height, mean (SD), cm & $168.03(10.27)$ \\
\hline Weight, mean (SD), kg & $79.72(20.60)$ \\
\hline Duration of $A D$, mean $(S D)$, years & $19.7(17.30)$ \\
\hline Age at $A D$ diagnosis, mean $(S D)$, years & $23.7(23.13)$ \\
\hline EASI, mean (SD) & $16.90(13.36)$ \\
\hline Peak Pruritus NRS, mean (SD) & $6.9(2.30)$ \\
\hline
\end{tabular}

\section{RESULTS (CONT.)}

Table 2. Family history of AD.

Patients with family history of $A D, n(\%)$

Relationship of family member who had AD, $n(\%)$

Mother

Father

Sibling

Other

$\mathbf{N}=\mathbf{3 1 5}$
$110(34.9)$

$40(12.7)$
$27(8.6)$
$41(13.0)$
$13(4.1)$
$32(10.2)$

Table 3. Proportion of patients with type 2 inflammatory comorbidities ${ }^{a}$ in the 12 months before dupilumab initiation

N $=315$

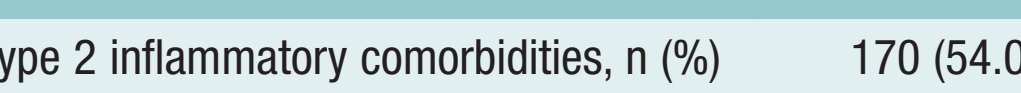

Allergic rhinitis

$\begin{array}{ll}\text { Chronic urticaria } & 17(5.4)\end{array}$

$=5 \%$ of patients.

$7(5.4)$

- The reported history of ocular conditions is shown in Table 4

Seasonal allergic conjunctivitis was most commonly $(19.0 \%)$ reported for a mean (SD) of $82.6(109.84)$ days in the past year

AD treatment history

$50.8 \%$ of the patients received $\geq 1$ topical and 1 systemic tion for $\mathrm{AD}$ (Table 5)

$41.0 \%$ used systemic corticosteroids; methotrexate $(10.2 \%)$ was the most commonly used systemic non-
steroidal immunosuppressant
Table 4. Ocular history over the past 12 months. ${ }^{a}$

\begin{tabular}{lcc|} 
& \multicolumn{2}{c}{$\mathbf{N}=\mathbf{3 1 5}$} \\
\cline { 2 - 3 } & $\mathbf{n}(\%)$ & $\begin{array}{c}\text { No. of days of } \\
\text { active condition, } \\
\text { mean (SD) }\end{array}$ \\
Seasonal allergic conjunctivitis & $60(19.0)$ & $82.6(109.84)$ \\
Dry eye & $29(9.2)$ & $121.3(149.01)$ \\
Perennial allergic conjunctivitis & $17(5.4)$ & $198.4(160.11)$ \\
Ophthalmic herpes simplex & $5(1.6)$ & $3.0(3.67)$ \\
Blepharitis & $4(1.3)$ & $89.0(141.59)$ \\
\hline "Conditions reported in $>$ 1\% of patients. & &
\end{tabular}

Table 5. AD treatment history (life-long recall).

N (\%)

$\geq 1$ prior AD medication 315 (100.0)

Previous use of $\geq 1$ topical (TC//TCS/PDE4

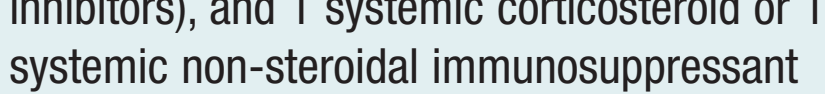

Previous use of systemic corticosteroids

Previous use of systemic non-steroida

Methotrexate

Cyclosporine

Mycophenolate

Azzathioprine $3(1.0)$

- In the year prior to enrollment, most patients used topical corticosteroids $(90.8 \%)$, followed by systemic

corticosteroids (36.2\%) and TCls (35.9\%) (Table 6)

The average duration of use for these medications was 178.9, 39.6, and 94.6 days, during the past year, respectively
Table 6. AD treatment history (12-month recall).

\begin{tabular}{lcc} 
& \multicolumn{2}{c}{$\mathbf{N}=\mathbf{3 1 5}$} \\
\cline { 2 - 3 }$\geq 1$ AD medication & $\mathbf{n}(\%)$ & $\begin{array}{c}\text { Mean number } \\
\text { of days }\end{array}$ \\
TCS & $294(93.3)$ & $\mathbf{N} / \mathbf{A}$ \\
TCI & $286(90.8)$ & 178.9 \\
PDE4 inhibitors & $113(35.9)$ & 94.6 \\
Phototherapy & $62(19.7)$ & 66.4 \\
Systemic corticosteroids & $23(7.3)$ & 59.7 \\
Systemic non-steroidal & $114(36.2)$ & 39.6 \\
immunosuppressants & $44(14.0)$ & 150.1 \\
$\quad$ Methotrexate & $24(7.6)$ & 111.6 \\
Cyclosporine & $22(7.0)$ & 160.5 \\
Mycophenolate & $6(1.9)$ & 40.5 \\
Azathioprine & $2(0.6)$ & 75.0 \\
\hline
\end{tabular}

Azathioprine

\section{CONCLUSIONS}

- Family history of AD was common in patients enrolled in this real-world registry

- Comorbid ocular diseases were frequently reported, including one-fifth of patients reporting seasonal allergic conjunctivitis

- All patients had received other AD treatments before initiating dupilumab, and almost al used $\geq 1$ AD medication in the past year

- Half of the patients in PROSE also received $\geq 1$ topical and $\geq 1$ systemic treatment during their life 